\title{
Percepción y hábitos de salud bucodental en niños y adolescentes de Navarra, 2007
}

\author{
Oral health perception and oral habits in children and teenagers \\ in Navarre, 2007
}

\author{
J. Artázcoz¹, F. J. Cortés, E. Rosel, P. González Rodríguez, M. Bravo
}

\section{RESUMEN}

Fundamento. Conocer la auto-percepción del nivel de salud oral y de las necesidades de tratamiento, los hábitos de higiene y la frecuencia de asistencia al dentista de los escolares de Navarra.

Material y métodos. La población de estudio fue la de 6, 12 y 14 años de edad. La muestra se obtuvo de los colegios de $1^{\circ}$ y $6^{\circ}$ de Enseñanza Primaria (EP) y $2^{\circ}$ de Enseñanza Secundaria Obligatoria (ESO) y la recogida de datos se hizo mediante cuestionarios auto-cumplimentados.

Resultados. El 92\%, a los 6 y 12 años, y el 82,9\% a los 14 años está satisfecho con su salud oral. El 13,1\% de los de 12 años y el 19,3\% de 14 están descontentos, sobre todo por el mal alineamiento dental. Aproximadamente, un tercio cree necesitar algún tratamiento, sobre todo obturaciones a los 6 años y ortodoncia a los 12 y 14 . Casi dos tercios dicen cepillarse los dientes más de una vez al día y entre el 80 y el $90 \%$ dicen haber ido al dentista en el último año. Más del $75 \%$ de los responsables de los escolares de 6 y 12 años valoran positivamente las prestaciones del Programa de Atención Dental Infantil (PADI) y más del 95\% está satisfecho con la asistencia que el dentista del PADI presta a sus hijos. El sexo, medio de residencia y nivel socioeconómico apenas influyen en la percepción de salud, frecuencia de cepillado o necesidades de tratamiento sentidas.

Conclusión. El estudio revela que la percepción subjetiva de salud dental es buena y que un tercio de los encuestados creen necesitar algún tipo de tratamiento. Es muy positivo que la mayoría digan que hayan ido al dentista en el último año y que se cepillan la boca más de una vez al día, mejorando los resultados de estudios anteriores.

Palabras clave. "Encuesta dental»; "Navarra"; "Servicios de salud dental»; "Atención dental infantil"; "Demanda y necesidades de servicios de salud".

\section{An. Sist. Sanit. Navar. 2010; 33 (1): 51-64}

1. Sección de Salud Bucodental. Servicio Navarro de Salud/Osasunbidea. Pamplona.

Recepción: 15 de mayo de 2009

Aceptación provisional: 1 de diciembre de 2009

Aceptación definitiva: 4 de febrero de 2010

Financiación

Proyecto financiado por el Departamento de Salud del Gobierno de Navarra y galardonado con la Beca Mikel Larumbe Zazu 2006 (Resolución 2131/2006, de 7 de noviembre, del Director General de Salud).

\begin{abstract}
Background. To determine the self-perception of oral health levels and the need for treatment, hygienic habits and frequency of visits to the dentist amongst schoolchildren in Navarre.
\end{abstract}

Methods. The population of the study was aged 6, 12 and 14 years. The sample was obtained from schools teaching $1^{\text {st }}$ and $6^{\text {th }}$ year in Primary Education and $2^{\text {nd }}$ year in Compulsory Secondary Education. The data was gathered through self-answering questionnaires.

Results. Ninety-two percent of 6 and 12 year olds, and $82.9 \%$ of 14 year olds are satisfied with their oral health. Thirteen point one percent of 12 year olds and $19.3 \%$ of 14 year olds are dissatisfied, above all with misaligned teeth. Approximately one-third think they need some treatment, above all fillings at age 6 and orthodontics at 12 and 14 years. Nearly two-thirds say they brush their teeth more than once a day and between 80 and $90 \%$ say they have visited the dentist in the last year. More than $75 \%$ of those responsible for the schoolchildren aged 6 and 12 years make a positive evaluation of the contributions of the child dental care program (PADI) and more than $95 \%$ are satisfied with the care given by PADI dentists to their children. Sex, average residence and socioeconomic level barely influence the perception of health, frequency of brushing teeth or need for treatment.

Conclusion. The study reveals that the subjective perception of oral health is good and that one-third of those surveyed think they need some type of treatment. It is very positive that they majority say that they have been to the dentist in the last year and that they brush their teeth more than once a day, which is an improvement on the results of previous studies.

Key words. Oral health survey. Navarre. Oral health services. Dental care for children. Health service needs and demand. 


\section{INTRODUCCIÓN}

Durante el año 2007 se llevó a cabo la $4^{a}$ Encuesta de Salud Dental de la población infantil de Navarra, cuyos datos clínicos son publicados en este mismo número de la revista ${ }^{1}$. Las anteriores datan de los años 1987, 1997 y 2002 y ofrecen, por tanto, la posibilidad de monitorizar la evolución en Navarra de esta área de la salud en los últimos 20 años, de sus hábitos y actitudes en salud oral ${ }^{2-4}$.

Todas las encuestas contemplaron una parte clínica de medición del estado de salud oral y una parte de cuestionario estructurado de preguntas para conocer los hábitos, conocimientos y actitudes en relación a la salud oral de esta población.

Los objetivos del presente trabajo están referidos a la población escolar de Navarra en 2007 y son:

- Conocer la auto-percepción del nivel de salud oral y de las necesidades de tratamiento.

- Conocer la frecuencia de asistencia al dentista así como la valoración, por parte de los padres/madres, del Programa de Atención Dental Infantil (PADI).

- Conocer los hábitos de higiene.

\section{POBLACIÓN Y MÉTODOS}

La descripción detallada de la metodología puede consultarse en "La salud dental de los niños y adolescentes de Navarra, $2007 \wedge^{1}$. He aquí un breve resumen: la población objeto de estudio ha estado constituida por los niños/as de Navarra de 6, 12 y 14 años de edad. La muestra se obtuvo, respectivamente, a partir del listado de de $1^{\circ}$ y $6^{\circ}$ de Enseñanza Primaria (EP) y $2^{\circ}$ de Enseñanza Secundaria Obligatoria (ESO) de los centros educativos del curso académico 2006-07; se realizó una selección polietápica al azar tomando como unidad de muestreo el aula, procediéndose a una selección principal y otra de reserva. Todos los niños/as de cada aula seleccionada eran objeto de estudio. El número de aulas seleccionadas se hizo en base a conseguir una muestra final para cada grupo de edad de, como mínimo, 400 escolares. Fueron seleccionados 28 centros de EP y 20 de ESO, repartidos por toda la geografía de la Comunidad Foral de Navarra.

Se definieron variables de estudio socio-demográficas [edad, sexo, residencia urbano/rural, nivel socio-económico (NSE) y origen español/extranjero] y variables de percepción del estado de salud oral, necesidades sentidas de tratamiento, de hábitos higiénicos y de utilización de servicios dentales.

El estudio se llevó a cabo en los propios centros educativos que aceptaron participar, durante los meses de abril y mayo de 2007 y una vez obtenido el consentimiento informado de los responsables de los escolares. Se diseñó un cuestionario auto-contestado, con preguntas cerradas y estructurado en tres áreas:

1. Auto-percepción de salud y necesidades sentidas.

2. Asistencia al dentista.

3. Hábitos higiénicos.

Las respuestas en el caso de auto-percepción variaban de "muy bueno" a "muy malo" y/o "muy satisfecho" a "muy insatisfecho". Los cuestionarios se acompañaron de una hoja de instrucciones.

En los escolares de $1^{\circ}$ y $6^{\circ}$ de EP los cuestionarios se enviaron a sus domicilios, dirigidos a los padres, para ser rellenados con la participación de su hijo/a; unas preguntas estaban dirigidas a los padres y otras directamente a los niños. En los de $2^{\circ}$ de ESO los cuestionarios fueron contestados directamente por los escolares.

Todo el material necesario junto con una hoja de instrucciones sobre su manejo fue enviado a los colegios participantes para su distribución: en las propias aulas en el caso de $2^{\circ}$ de ESO, destinando un tiempo específico para ello y en los domicilios de los participantes en el caso de $1^{\circ}$ y $6^{\circ}$ de EP.

Los datos obtenidos fueron informatizados con el programa Access XP 2003 (10.6771.6811) SP3, con un sistema automá- 
tico de detección de errores, y el análisis estadístico se realizó con el programa SPSS Windows v.15.0 (SPSS Inc., Chicago, II). Se calcularon valores descriptivos e intervalos de confianza. Las pruebas de asociación se indican a pie de cada tabla.

\section{RESULTADOS}

La tasa de respuesta de la muestra de aulas inicialmente seleccionada fue del 97,54\%. El 2,46\% de rechazo corresponde a uno de los centros que rehusó participar en el estudio, por lo que fue sustituido por otro de reserva. Respecto al número de cuestionarios recibidos de vuelta, su tasa de respuesta fue del $83,9 \%, 91,2$ y $96,4 \%$ en los tres cursos de $1^{\circ}, 6^{\circ}$ de EP y $2^{\circ}$ de ESO, respectivamente.

La tabla 1 describe la muestra por edad y variables socio-demográficas; el $89,2 \%$ es de origen nacional y el $10,8 \%$ de origen extranjero. Las medias de edad resultantes fueron $6,35,11,42$ y 13,47 años. Por ello, en las cabeceras de las tablas se indican los intervalos de edad 6-7, 11-12 y 13-14, aunque en el texto, para abreviar, se mencionan los grupos referencia 6, 12 y 14 .

Tabla 1. Muestra de niños de $1^{\circ}$ y $6^{\circ}$ de EP y de $2^{\circ}$ de ESO.

\begin{tabular}{|c|c|c|c|c|c|c|c|c|}
\hline & \multicolumn{2}{|c|}{ 6-7 } & \multicolumn{2}{|c|}{ 11-12 } & \multicolumn{2}{|c|}{$13-14$} & \multicolumn{2}{|c|}{ Total } \\
\hline & $\mathbf{n}$ & $\%$ & $\mathbf{n}$ & $\%$ & $\mathbf{n}$ & $\%$ & $\mathbf{n}$ & $\%$ \\
\hline \multicolumn{9}{|l|}{ Edad } \\
\hline 6 años ( $\left.1^{\circ} \mathrm{EP}\right)$ & 339 & 65,1 & 0 & - & 0 & - & 339 & 24,3 \\
\hline 7 años ( $\left.1^{\rho} \mathrm{EP}\right)$ & 182 & 34,9 & 0 & - & 0 & - & 182 & 13,0 \\
\hline 11 años ( $\left.6^{\circ} \mathrm{EP}\right)$ & 0 & - & 286 & 58,6 & 0 & - & 286 & 20,5 \\
\hline 12 años ( $\left.6^{\circ} \mathrm{EP}\right)$ & 0 & - & 202 & 41,4 & 0 & - & 202 & 14,5 \\
\hline 13 años ( $2^{\circ}$ ESO) & 0 & - & 0 & - & 207 & 53,4 & 207 & 14,7 \\
\hline 14 años ( $2^{\circ}$ ESO) & 0 & - & 0 & - & 181 & 46,6 & 181 & 13,0 \\
\hline Edad media $( \pm$ de $)$ & \multicolumn{2}{|c|}{$6,35 \pm 0,48$} & \multicolumn{2}{|c|}{$11,42 \pm 0,49$} & \multicolumn{2}{|c|}{$13,47 \pm 0,50$} & & \\
\hline Hombre & 269 & 51,6 & 250 & 51,2 & 198 & 51,0 & 717 & 51,3 \\
\hline Mujer & 252 & 48,4 & 238 & 48,8 & 190 & 49,0 & 680 & 48,7 \\
\hline Urbano & 385 & 73,9 & 347 & 71,1 & 267 & 68,8 & 999 & 71,5 \\
\hline Rural & 136 & 26,1 & 141 & 28,9 & 121 & 31,2 & 368 & 28,5 \\
\hline NSE Alto & 124 & 24,4 & 141 & 29,0 & 115 & 29,9 & 380 & 27,6 \\
\hline NSE Medio & 119 & 23,4 & 138 & 28,4 & 118 & 30,6 & 375 & 27,2 \\
\hline NSE Bajo & 265 & 52,2 & 207 & 42,6 & 152 & 39,5 & 624 & 45,3 \\
\hline Perdidos & 13 & & 2 & & 3 & & 18 & \\
\hline Origen España & 448 & 86,0 & 449 & 92,0 & 349 & 89,9 & 1.246 & 89,2 \\
\hline Origen extranjero & 73 & 14,0 & 39 & 8,0 & 39 & 10,1 & 151 & 10,8 \\
\hline Unión Europea* & 7 & 9,6 & 6 & 15,4 & 3 & 7,7 & 16 & 10,6 \\
\hline Otro país Europa* & 11 & 15,1 & 8 & 20,5 & 4 & 10,3 & 23 & 15,9 \\
\hline Canadá o USA* & 0 & - & 1 & 2,6 & 0 & - & 1 & 0,7 \\
\hline Otro país América* & 46 & 63,0 & 21 & 53,7 & 30 & 76,9 & 97 & 64,2 \\
\hline Asia* & 2 & 2,7 & 2 & 5,1 & 0 & - & 4 & 2,6 \\
\hline África* & 7 & 9,6 & 1 & 2,6 & 2 & 5,1 & 10 & 6,6 \\
\hline Total & 521 & & 488 & & 388 & & 1.397 & \\
\hline
\end{tabular}

* Se presenta el porcentaje de individuos de cada posible origen extranjero con respecto al total de extranjeros. 


\section{Salud percibida}

A los 6 y 12 años, el 77-78\% de los padres perciben la salud oral de sus hijos como buena o muy buena, el $20 \%$ como regular y el $2-4 \%$ como mala o muy mala. La percepción es más positiva cuando la pregunta va dirigida a los propios escolares: el $92 \%$ está satisfecho o muy satisfecho, mientras que el 8\% está bastante o muy insatisfecho. Respecto de la apariencia de sus dientes, cabe reseñar que, a los 12 años, el $13,1 \%$ dice estar descontento o muy descontento, y entre los motivos del descontento el $72,4 \%$ es por no estar bien alineados y el $27,6 \%$ por no gustarles la forma o el tamaño de sus dientes (Tablas 2 y 3 ).

Tabla 2. Salud percibida y necesidades de tratamiento sentidas a los 6 años. Total y por NSE (nivel socio-económico).

\begin{tabular}{cccccc}
\multicolumn{3}{c}{ Total 6 años } & \multicolumn{3}{c}{ NSE } \\
\hline n & $\%$ & IC-95\% & Alto & Medio & Bajo
\end{tabular}

\section{En general, diría que la salud bucodental de su hijo/a es: (señale una)}

$\begin{array}{lrrrrrr}\text { 1. Muy buena } & 70 & 16,1 & (12,6-19,5) & 17,2 & 18,1 & 14,0 \\ \text { 2. Buena } & 264 & 60,5 & (55,9-65,2) & 60,4 & 66,3 & 58,0 \\ \text { 3. Regular } & 83 & 19,0 & (15,3-22,7) & 18,1 & 13,8 & 22,0 \\ \text { 4. Mala } & 17 & 3,9 & (2,1-5,7) & 4,3 & 0,9 & 5,5 \\ \text { 5. Muy mala } & 2 & 0,5 & (0,0-1,1) & 0,0 & 0,9 & 0,5 \\ \text { No sabe, no contesta } & 1 & - & - & & & \end{array}$

2. ¿Su hijo/a está satisfecho o contento con su salud bucodental? (señalar una)

$\begin{array}{lrrrrrr}\text { 1. Muy satisfecho } & 120 & 28,0 & (23,7-32,2) & 26,3 & 28,9 & 28,3 \\ \text { 2. Bastante satisfecho } & 276 & 64,3 & (59,8-68,9) & 69,3 & 65,8 & 60,6 \\ \text { 3. Bastante insatisfecho } & 28 & 6,5 & (4,2-8,9) & 4,4 & 5,3 & 8,6 \\ \text { 4. Muy insatisfecho } & 5 & 1,2 & (0,1-2,2) & 0,0 & 0,0 & 2,5 \\ \text { No sabe, no contesta } & 8 & - & - & & & \end{array}$

3. ¿Su hijo/a está satisfecho o contento con la apariencia de sus dientes? (señalar una)

$\begin{array}{lrrrrrr}\text { 1. Muy contento (pase a la 5) } & 137 & 31,9 & (27,4-36,3) & 28,7 & 35,3 & 30,6 \\ \text { 2. Bastante contento (pase a la 5) } & 163 & 37,9 & (33,3-42,5) & 43,5 & 36,2 & 36,2 \\ \text { 3. Ni contento ni descontento (pase a la 5) } & 115 & 26,7 & (22,5-30,9) & 26,1 & 25,0 & 28,6 \\ \text { 4. Descontento (pase a la 4) } & 10 & 2,3 & (0,9-3,8) & 1,7 & 2,6 & 2,6 \\ \text { 5. Muy descontento (pase a la 4) } & 5 & 1,2 & (0,1-2,2) & 0,0 & 0,9 & 2,0 \\ \text { No sabe, no contesta } & 7 & - & - & & & \end{array}$

4. Si su hijo/a está descontento o muy descontento, indicar el motivo (señalar 1 o más) ${ }^{1}$
1. No están bien alineados
$9 \quad 60,0 \quad(31,9-88,1)$
2. No le gusta el color
$1 \quad 6,7 \quad(0,0-21,0)$
3. No le gusta la forma o el tamaño
$4 \quad 26,7 \quad(1,3-52,0)$
4. No le gusta mucho pero no le importa demasiado
$1 \quad 6,7 \quad(0,0-21,0)$
5. Otro
$4 \quad 26,7 \quad(1,3-52,0)$ 


\begin{tabular}{lrrrrrrr}
\hline & \multicolumn{3}{c}{ Total 6 años } & \multicolumn{3}{c}{ NSE } \\
\cline { 2 - 7 } & \multicolumn{1}{c}{$\mathbf{n}$} & $\%$ & \multicolumn{1}{c}{ IC-95\% } & Alto & Medio & Bajo \\
\hline 9. En estos momentos, isu hijo/a cree que necesita algún tipo de tratamiento dental? (señale una) & \\
1. Sí (pase a la 10) & 95 & 22,2 & $(18,2-26,1)$ & 23,7 & 11,3 & $27,6^{*}$ \\
2. No (pase a la 11) & 258 & 60,3 & $(55,6-64,9)$ & 64,9 & 68,7 & 52,5 \\
3. No sé (pase a la 11) & 75 & 17,5 & $(13,9-21,1)$ & 11,4 & 20,0 & 19,9 \\
No contesta & 9 & - & & & & \\
\hline
\end{tabular}

10. ¿Qué tipo de tratamiento cree su hijo/a que necesita? (señale 1 o más)
1. Quitar el dolor
$7 \quad 7,5$
$(2,1-13,0)$
2. Empaste
$45 \quad 48,4 \quad(38,0-58,7)$
3. Sacar una muela
$9 \quad 9,7 \quad(3,6-15,8)$
4. Aparato de ortodoncia
$33 \quad 35,5 \quad(25,6-45,4)$
5. Otro tratamiento
$16 \quad 17,2 \quad(9,4-25,0)$
No sabe, no contesta
2

${ }^{*} \mathrm{p}<0,05$

Las preguntas multirrespuestas no suman $100 \%$.

1. Los 15 individuos que respondieron a los ítems 4 y 5 de la pregunta 3 contestaron a la pregunta 4

2. De los 95 individuos que respondieron el ítem 1 de la pregunta 9 , sólo 93 contestaron a la pregunta 10.

Tabla 3. Salud percibida y necesidades de tratamiento sentidas a los 12 años. Total y por NSE (nivel socio-económico).

\begin{tabular}{cccccc} 
& \multicolumn{2}{l}{ Total 12 años } & \multicolumn{3}{c}{ NSE } \\
\hline n & $\%$ & IC-95\% & Alto & Medio & Bajo
\end{tabular}

\section{En general, dirías que tu salud bucodental de su hijo/a es: (señala una)}

$\begin{array}{lcccccc}\text { 1. Muy buena } & 56 & 12,6 & (9,5-15,7) & 13,2 & 9,8 & 14,5 \\ \text { 2. Buena } & 289 & 65,3 & (60,8-69,7) & 66,2 & 71,4 & 59,9 \\ \text { 3. Regular } & 90 & 20,3 & (16,6-24,1) & 20,6 & 18,0 & 21,5 \\ \text { 4. Mala } & 8 & 1,8 & (0,6-3,1) & 0,0 & 0,8 & 4,1 \\ \text { 5. Muy mala } & 0 & 0 & - & 0,0 & 0,0 & 0,0 \\ \text { No sabe, no contesta } & 2 & - & - & & & \end{array}$

\section{2. ¿Su hijo/a está satisfecho o contento con su salud bucodental? (señalar una)}

\begin{tabular}{lcccccc} 
1. Muy satisfecho & 101 & 22,9 & $(18,9-26,8)$ & 21,5 & 22,6 & 23,8 \\
2. Bastante satisfecho & 306 & 69,2 & $(64,9-73,6)$ & 68,9 & 72,9 & 66,9 \\
3. Bastante insatisfecho & 31 & 7,0 & $(4,6-9,4)$ & 8,1 & 4,5 & 8,1 \\
4. Muy insatisfecho & 4 & 0,9 & $(0,0-1,8)$ & 1,5 & 0,0 & 1,2 \\
No sabe, no contesta & 3 & - & - & & & \\
\hline
\end{tabular}




\begin{tabular}{lcccccc}
\hline & \multicolumn{3}{c}{ Total 12 años } & \multicolumn{3}{c}{ NSE } \\
\cline { 2 - 7 } & $\mathbf{n}$ & $\%$ & IC-95\% & Alto & Medio & Bajo \\
\hline 3. ¿Su hijo/a está satisfecho o contento con la apariencia de sus dientes? (señalar una) & $\mathbf{1}$ \\
1. Muy contento (pase a la 5) & 85 & 19,3 & $(15,6-23,0)$ & 19,4 & 15,0 & 22,2 \\
2. Bastante contento (pase a la 5) & 155 & 35,3 & $(30,7-39,7)$ & 38,0 & 43,6 & 26,9 \\
3. Ni contento ni descontento (pase a la 5) & 142 & 32,3 & $(27,9-36,7)$ & 28,4 & 30,9 & 36,8 \\
4. Descontento (pase a la 4) & 53 & 12,0 & $(9,0-15,1)$ & 12,7 & 9,0 & 13,5 \\
5. Muy descontento (pase a la 4) & 5 & 1,1 & $(0,1-2,1)$ & 1,5 & 1,5 & 0,6 \\
No sabe, no contesta & 5 & - & - & & & \\
\hline
\end{tabular}

4. Si su hijo/a está descontento o muy descontento, indicar el motivo (señalar 1 o más)
1. No están bien alineados
$42 \quad 72,4$
$(60,6-84,3)$
2. No me gusta el color
$11 \quad 19,0$
$(8,6-29,4)$
3. No me gusta la forma o el tamaño
$16 \quad 27,6$
$(15,7-39,4)$
4. No me gusta mucho pero no me importa
$6 \quad 10,3$
$(2,3-18,4)$ demasiado

5. Otro $\quad 3 \quad 5,2 \quad(0,0-11,0)$

9. En estos momentos, ¿su hijo/a cree que necesita algún tipo de tratamiento dental? (señale u una) ${ }^{2}$

$\begin{array}{lcccccc}\text { 1. Sí (pasa a la pregunta 10) } & 160 & 36,3 & (31,8-40,8) & 37,5 & 42,7 & 30,8 \\ \text { 2. No (pasa a la pregunta 11) } & 237 & 53,7 & (49,1-58,4) & 52,2 & 48,9 & 58,7 \\ \text { 3. No sé (pasa a la pregunta 11) } & 44 & 10,0 & (7,2-12,8) & 10,3 & 8,4 & 10,5 \\ \text { No contesto } & 4 & & & & \end{array}$

10. ¿Qué tipo de tratamiento cree su hijo/a que necesita? (señale 1 o más)

$\begin{array}{lccc}\text { 1. Quitar el dolor } & 2 & 1,3 & (0,0-3,1) \\ \text { 2. Empaste } & 16 & 10,3 & (5,5-15,2) \\ \text { 3. Sacar una muela } & 7 & 4,5 & (1,2-7,8) \\ \text { 4. Aparato de ortodoncia } & 135 & 87,1 & (81,8-92,4) \\ \text { 5. Otro tratamiento } & 6 & 3,9 & (0,8-6,9) \\ \text { No sabe, no contesta } & 5 & - & -\end{array}$

Las preguntas multirrespuesta no suman $100 \%$.

1. Los 58 individuos que respondieron a los ítems 4 y 5 de la pregunta 3 contestaron a la pregunta 4 .

2. De los 160 individuos que respondieron el ítem 1 de la pregunta 9,155 contestaron a la pregunta 10.

A estas edades no hay diferencias significativas por nivel socio-económico en ninguno de los aspectos estudiados con relación a la percepción subjetiva del nivel de salud bucodental (Tablas 2 y 3 ). Tampoco aparecen diferencias significativas por sexo o residencia (datos no mostrados).

A los 14 años, el 82,9\% percibe su salud como buena o muy buena, el $14,4 \%$ como regular y el $2,7 \%$ como mala; en cuanto al nivel de satisfacción, el 6,4\% dicen estar descontentos o muy descontentos con su salud bucodental. Respecto de la apariencia de sus dientes, el 19,3\% dice estar descontento o muy descontento y entre las razones del descontento, el $57,7 \%$ por no estar bien alineados y el $43,7 \%$ por su color (Tabla 4). 
Tabla 4. Salud percibida y necesidades de tratamiento sentidas a los 14 años. Total y por NSE (nivel socio-económico).

\begin{tabular}{lcccccc}
\hline & \multicolumn{3}{c}{ Total 14 años } & \multicolumn{3}{c}{ NSE } \\
\cline { 2 - 8 } & $\mathbf{n}$ & \% & IC-95\% & Alto & Medio & Bajo \\
\hline 1. En general, dirías que tu salud bucodental es: (señala una) & & & & \\
1. Muy buena & 20 & 5,3 & $(3,1-7,6)$ & 9,6 & 3,4 & $3,6^{*}$ \\
2. Buena & 109 & 29,1 & $(24,5-33,8)$ & 30,8 & 33,1 & 25,2 \\
3. Regular & 181 & 48,5 & $(43,3-53,5)$ & 45,6 & 49,1 & 48,9 \\
4. Mala & 54 & 14,4 & $(10,9-18,0)$ & 10,5 & 11,0 & 20,9 \\
5. Muy mala & 10 & 2,7 & $(1,0-4,3)$ & 3,5 & 3,4 & 1,4 \\
\hline 2. ¿Estás satisfecho o contento con tu salud bucodental? (señala una) & & & \\
1. Muy contento & 67 & 17,9 & $(14,0-21,8)$ & 24,6 & 13,6 & $16,5^{*}$ \\
2. Bastante contento & 148 & 39,6 & $(34,6-44,6)$ & 41,2 & 47,4 & 30,2 \\
3. Ni contento ni descontento & 135 & 36,1 & $(31,2-44,0)$ & 28,1 & 31,4 & 47,6 \\
4. Descontento & 18 & 4,8 & $(2,6-7,0)$ & 2,6 & 6,8 & 5,0 \\
5. Muy descontento & 6 & 1,6 & $(0,3-2,9)$ & 3,5 & 0,8 & 0,7 \\
\hline 3. ¿Estás satisfecho o contento con la apariencia de tus dientes? (señala una) & & & \\
1. Muy contento (pase a la 5) & 58 & 15,7 & $(12,0-19,4)$ & 18,6 & 18,1 & 11,7 \\
2. Bastante contento (pase a la 5) & 105 & 28,5 & $(23,8-33,1)$ & 25,7 & 25,9 & 32,1 \\
3. Ni contento ni descontento (pase a la 5) & 135 & 36,5 & $(31,6-41,5)$ & 37,2 & 35,3 & 38,0 \\
4. Descontento (pase a la 4) & 60 & 16,3 & $(12,5-20,0)$ & 15,0 & 16,4 & 16,7 \\
5. Muy descontento (pase a la 4) & 11 & 3,0 & $(1,2-4,7)$ & 3,5 & 4,3 & 1,5 \\
No sabe, no contesta & 5 & - & - & & & \\
\hline 4. Si estas descyyyyyyyyyyyyyyy
\end{tabular}

4. Si estás descontento o muy descontento, indica el motivo (señala 10 más)

1. No están bien alineados $\quad 41 \quad 57,7 \quad(46,6-69,5)$

2. No me gusta el color $\quad 31 \quad 43,7 \quad(31,8-55,5)$

3. No me gusta la forma o el tamaño $\quad 19 \quad 26,8 \quad(16,2-37,3)$

4. No me gusta mucho pero no me importa $\quad 5 \quad 7,0 \quad(0,9-13,1)$

demasiado
5. Otro
$13 \quad 18,3$
$(9,1-27,5)$

\section{En estos momentos, ¿crees que necesitas algún tratamiento dental? (señala una) ${ }^{2}$}

$\begin{array}{lcccccc}\text { 1. Sí (pasa a la pregunta 8) } & 110 & 29,8 & (25,1-34,5) & 31,0 & 29,3 & 29,9 \\ \text { 2. No (pasa a la pregunta 9) } & 147 & 39,8 & (34,8-44,9) & 43,3 & 39,7 & 37,3 \\ \text { 3. No sé (pasa a la pregunta 9) } & 112 & 30,4 & (25,6-35,1) & 25,7 & 31,0 & 32,8 \\ \text { No contesto } & 5 & & & & & \end{array}$

\section{8. ¿Qué tipo de tratamiento crees que necesitas? (señala 1 o más)}

1. Quitar el dolor

$4 \quad 3,8 \quad(0,9-4,0)$

2. Empaste

$13 \quad 12,4 \quad(6,0-18,8)$

3. Sacar una muela

$3 \quad 2,9 \quad(0,0-6,1)$

4. Aparato de ortodoncia

$79 \quad 75,2 \quad(66,8-83,6)$

5. Otro tratamiento

No sabe, no contesta

$22 \quad 21,0 \quad(13,0-28,9)$

${ }^{*} \mathrm{p}<0,05$

Las preguntas multirrespuesta no suman $100 \%$.

1. Los 71 individuos que respondieron a los ítems 4 y 5 de la pregunta 3 contestaron a la pregunta 4 .

2. De los 110 individuos que respondieron al ítem 1 de la pregunta 7 , sólo 105 contestaron a la pregunta 8. 
A esta edad, los pertenecientes a nivel socioeconómico NSE bajo (NSE) califican peor su salud bucodental y están menos satisfechos con la misma $(\mathrm{p}<0,05)$ (Tabla 4). Sin embargo, ni sexo ni residencia generan diferencias significativas en su percepción de salud (datos no mostrados).

\section{Necesidades sentidas de tratamiento}

El 22,2\% de escolares de 6 años y el $36,3 \%$ de 12 creen que necesitan algún tipo de tratamiento. De ellos, a los 6 años, la mayoría cree que necesita un empaste $(48,4 \%)$ y/o un aparato de ortodoncia $(35,5 \%)$, mientras que entre los de 12 años la mayoría cree que necesita tratamiento de ortodoncia $(87,1 \%)$ y sólo el $10,3 \%$ empaste. Entre los de 6 años, el 7,5\% dice necesitar tratamiento por tener dolor (Tablas 2 y 3 ).

En cuanto al análisis de esta variable según el NSE al que se pertenece, los es- colares de 6 años de bajo NSE sienten más necesidades de tratamiento $(\mathrm{p}<0,05)$ que los de nivel alto y medio. Sin embargo, a los 12 años no aparecen diferencias significativas (Tablas 2 y 3 ).

A los 14 años, el 29,8\% cree tener alguna necesidad de tratamiento. De ellos, el $75,2 \%$ tratamiento de ortodoncia y el $12,4 \%$ empaste. No aparecen diferencias significativas por el NSE al que se pertenece (Tabla 4).

Tampoco aparecen diferencias significativas por sexo o por residencia en ninguno de los tres grupos de edad estudiados (datos no mostrados).

\section{Hábitos higiénicos}

A los 6 y 12 años, se cepillan los dientes más de una vez al día el 61,1 y $67,6 \%$ respectivamente y lo hacen sólo ocasionalmente el 7,5\% y 6,8\% (Tabla 5).

Tabla 5. Hábitos higiénicos, frecuencia según edad.

\begin{tabular}{|c|c|c|c|c|c|c|c|}
\hline & & \multicolumn{2}{|r|}{ 6-7 } & \multicolumn{2}{|c|}{$11-12$} & \multicolumn{2}{|c|}{$13-14$} \\
\hline & & $\%$ & IC-95\% & $\%$ & IC-95\% & $\%$ & IC-95\% \\
\hline \multirow{6}{*}{ 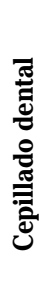 } & 1. Más de 1 vez al día & 61,1 & $(56,5-65,7)$ & 67,6 & $(63,3-72,0)$ & 63,3 & $(58,4-68,2)$ \\
\hline & 2. Una vez al día & 30,4 & $(26,1-34,8)$ & 23,3 & $(19,3-27,3)$ & 25,2 & $(20,8-29,6)$ \\
\hline & 3. Menos de una vez al día & 1,6 & $(0,4-2,8)$ & 2,9 & $(1,4-4,5)$ & 2,1 & $(0,7-3,6)$ \\
\hline & 4. De vez en cuando & 6,7 & $(4,3-9,0)$ & 5,7 & $(3,5-7,8)$ & 7,8 & $(5,0-10,5)$ \\
\hline & 5. Nunca/casi nunca & 0,2 & $(0,0-0,7)$ & 0,5 & $(0,0-1,1)$ & 1,6 & $(0,3-2,9)$ \\
\hline & No sabe, no contesta & - & - & - & - & - & - \\
\hline \multirow{5}{*}{ 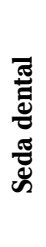 } & 1. Todos los días & - & - & - & - & 3,8 & $(1,8-5,7)$ \\
\hline & 2. De vez en cuando & - & - & - & - & 22,1 & $(17,9-26,3)$ \\
\hline & 3. Nunca-casi nunca & - & - & - & - & 66,6 & $(61,8-71,3)$ \\
\hline & 4. No se qué es la seda dental & - & - & - & - & 7,5 & $(4,8-10,2)$ \\
\hline & No sabe, no contesta & - & - & - & - & - & - \\
\hline
\end{tabular}


Respecto al análisis por variables sociodemográficas, sólo se apreciaron diferencias significativas $(\mathrm{p}<0,05)$ por sexo; concretamente, las chicas de 12 años que se cepillan más de una vez al día son más que los chicos (dato no mostrado).

A los 14 años, el 63,3\% dice cepillarse más de una vez al día y el 9,4\% sólo ocasionalmente. A esta edad utilizan seda dental a diario el 3,8\% y de vez en cuando el $25,2 \%$ (Tabla 5).
En cuanto al análisis por variables socio-demográficas, a los 14 años aparece alguna diferencia significativa por sexo, medio de residencia y origen. Son más las chicas y los residentes en medio rural los que se cepillan los dientes más de una vez $(\mathrm{p}<0,05)$, mientras que los extranjeros se cepillan a diario con más frecuencia que los de origen España $(\mathrm{p}<0,05)$. Sin embargo el análisis por NSE no refleja ninguna diferencia significativa en ninguno de los items estudiados (Tabla 6).

Tabla 6. Frecuencia de cepillado dental a los 14 años, según variables sociodemográficas (en el interior de la tabla se dan porcentajes que suman siempre $100 \%$ para cada categoría).

\begin{tabular}{lcccccccccc}
\hline & \multicolumn{2}{c}{ Sexo } & \multicolumn{2}{c}{ Origen } & \multicolumn{2}{c}{ Residencia } & \multicolumn{3}{c}{ NSE $^{\text {a }}$} \\
\cline { 2 - 10 } & Hombre & Mujer & España & Extranjero & Urbano & Rural & Alto & Medio & Bajo \\
\hline Más de 1 vez al día & 53,6 & $73,5^{*}$ & 60,8 & $87,9^{*}$ & 68,4 & $52,1^{*}$ & 64,6 & 66,9 & 59,0 \\
Una vez al día & 30,8 & 9,2 & 27,1 & $6,1^{*}$ & 21,0 & 34,2 & 23,9 & 22,2 & 28,8 \\
Menos de una vez & 2,6 & 1,7 & 2,1 & 3,0 & 2,0 & 2,6 & 1,8 & 2,5 & 2,2 \\
al día & & & & & & & & & \\
De vez en cuando & 10,4 & 5,0 & 8,2 & 3,0 & 7,0 & 9,4 & 8,8 & 5,9 & 8,6 \\
Nunca/casi nunca & 2,6 & 0,6 & 1,8 & 0 & 1,6 & 1,7 & 0,9 & 2,5 & 1,4 \\
\hline
\end{tabular}

a: Nivel socio-económico

${ }^{*} \mathrm{p}<0,05$

\section{Asistencia al dentista en el último año}

A los 6 y 12 años, el 87,4 y 91,6\% de los padres afirma haber llevado a su hijo al dentista en el último año. A un dentista del PADI dicen haberlo llevado el 82,7 y el $83,7 \%$, respectivamente (Tabla 7 ). No hay diferencias significativas por las variables socio-demográficas de sexo y medio de residencia, pero sí por NSE; acuden más los de nivel alto $(\mathrm{p}<0,05)$. Asimismo, apa- recen diferencias significativas $(\mathrm{p}<0,05)$ por origen, acudiendo menos los de origen extranjero, en los tres grupos de edad (Tabla 8).

A los 14 años, el 82,6\% dice haber ido al dentista en el último año y, de ellos, el $51,5 \%$ a un dentista PADI y el $31,1 \%$ a uno privado (Tabla 7). La única diferencia significativa por variables socio-demográficas aparece según origen $(\mathrm{p}<0,05)$, acudiendo menos los de origen extranjero (Tabla 8). 
Tabla 7. Asistencia al dentista en el último año según edad.

\begin{tabular}{|c|c|c|c|c|c|c|}
\hline & \multicolumn{2}{|c|}{ 6-7 } & \multicolumn{2}{|c|}{$11-12$} & \multicolumn{2}{|c|}{$13-14$} \\
\hline & $\%$ & IC-95\% & $\%$ & IC-95\% & $\%$ & IC-95\% \\
\hline No va & 12,6 & $(9,5-15,8)$ & 8,4 & $(5,8-11,0)$ & 17,4 & $(13,5-21,3)$ \\
\hline Dentista privado & 4,7 & $(2,7-6,7)$ & 7,9 & $(5,4-10,4)$ & 31,1 & $(26,3-35,8)$ \\
\hline Dentista del PADI* & 82,7 & $(79,1-86,3)$ & 83,7 & $(80,3-87,2)$ & 51,5 & $(46,4-56,6)$ \\
\hline
\end{tabular}

* Programa de Atención Dental Infantil y Juvenil.

Tabla 8. Asistencia al dentista en el último año según sexo, medio de residencia, origen, nivel socioeconómico y año (en el interior de la tabla se dan porcentajes que suman siempre 100\%).

\begin{tabular}{lccccccccc}
\hline & \multicolumn{3}{c}{ 6-7 años } & \multicolumn{3}{c}{ 11-12 años } & \multicolumn{3}{c}{ 13-14 años } \\
\cline { 2 - 10 } & No va & Privado & PADI & No va & Privado & PADI & No va & Privado & PADI \\
\hline Hombre & 13,0 & 4,2 & 82,8 & 10,6 & 6,0 & 83,4 & 19,7 & 31,9 & 48,4 \\
Mujer & 12,2 & 5,2 & 82,6 & 6,2 & 9,8 & 84,0 & 15,1 & 30,2 & 54,7 \\
\hline Urbano & 12,8 & 6,1 & 81,2 & 7,9 & 9,1 & 83,0 & 19,1 & 31,5 & 49,4 \\
Rural & 12,2 & 0,9 & 87,0 & 9,7 & 4,8 & 85,5 & 13,8 & 30,2 & 56,0 \\
\hline Español & 9,5 & 3,4 & 87,1 & 6,1 & 7,1 & 86,8 & 15,0 & 32,9 & 52,1 \\
Extranjero & $37,5^{*}$ & $14,6^{*}$ & $47,9^{*}$ & $36,4^{*}$ & $18,2^{*}$ & $45,4^{*}$ & $42,4^{*}$ & $12,1^{*}$ & 45,5 \\
\hline NSE Alto & 8,8 & 3,5 & $87,7^{*}$ & 8,9 & 12,6 & $78,5^{*}$ & 17,5 & 35,1 & 47,4 \\
NSE Medio & 9,5 & 1,7 & 88,8 & 2,3 & 6,1 & 91,6 & 18,3 & 36,5 & 45,2 \\
NSE Bajo & 16,4 & 7,1 & 76,5 & 12,7 & 5,8 & 81,5 & 16,3 & 23,0 & 60,7 \\
\hline 1997 $^{*}$ * & 28,9 & 20,8 & 50,3 & 17,5 & 21,3 & 61,2 & 32,8 & 67,2 & \\
2002 $^{\text {a* * }}$ & 16,3 & 11,5 & 72,2 & 8,8 & 19,4 & 71,8 & 11,8 & $88,2^{\text {b }}$ & \\
2007 $^{*}$ * & 12,6 & 4,7 & 82,7 & 8,4 & 7,9 & 83,7 & 17,4 & 31,1 & 51,5 \\
\hline
\end{tabular}

PADI: Programa de Atención Dental Infantil y Juvenil

*: $\mathrm{p}<0,05$ (test chi cuadrado)

Las comparaciones entre las categorías sexo y residencia no son significativas.

a: Los datos para 2002 están tomados de la muestra de origen España.

b: El dato no diferencia si acude a privado o PADI. Se han colapsado las categorías Privado y PADI.

** Hay asociación significativa ( $\mathrm{p}<0,001$, con chi cuadrado) entre el año y las categorías de asistencia al dentista.

\section{Valoración del PADI por los padres}

El 74,7 y el $79,8 \%$, respectivamente, de los responsables de los escolares de 6 y 12 años valoran las prestaciones del PADI como bien o muy bien. El 19,9 y 17,2\% como regular y el 5,4 y 3,0\% como mal o muy mal.
A esas mismas edades, y con relación a la asistencia que el dentista PADI presta a sus hijos, el 95,7 y $96,4 \%$ de los padres/ madres está bastante o muy satisfecho y el 4,3 y $3,6 \%$ bastante o muy insatisfecho (Tabla 9). 
Tabla 9. Valoración del programa de atención dental infantil por parte de los padres.

\begin{tabular}{|c|c|c|c|c|c|c|}
\hline & \multicolumn{3}{|r|}{ 6-7 } & \multicolumn{3}{|c|}{ 11-12 } \\
\hline & $\mathbf{n}$ & $\%$ & IC-95\% & $\mathbf{n}$ & $\%$ & IC-95\% \\
\hline \multicolumn{7}{|c|}{ ¿Cómo valora Ud. las prestaciones que cubre el PADI*? (señale una) } \\
\hline 1. Muy bien & 93 & 26,4 & $(21,8-31,0)$ & 112 & 30,6 & $(25,9-35,3)$ \\
\hline 2. Bien & 170 & 48,3 & $(43,0-53,5)$ & 180 & 49,2 & $(44,0-54,3)$ \\
\hline 3. Regular & 70 & 19,9 & $(15,7-24,1)$ & 63 & 17,2 & $(13,3-21,1)$ \\
\hline 4. Mal & 16 & 4,5 & $(2,4-6,7)$ & 8 & 2,2 & $(0,7-3,7)$ \\
\hline 5. Muy mal & 3 & 0,9 & $(0,0-1,8)$ & 3 & 0,8 & $(0,0-1,7)$ \\
\hline No sabe, no contesta & 2 & - & - & 4 & - & - \\
\hline \multicolumn{7}{|c|}{ En general, ¿está satisfecho/a con la asistencia que el dentista del PADI le presta a su hijo/a? (señale una) } \\
\hline 1. Muy satisfecho & 231 & 65,6 & $(60,6-70,6)$ & 246 & 67,7 & $(62,9-72,6)$ \\
\hline 2. Bastante satisfecho & 106 & 30,1 & $(25,3-34,9)$ & 104 & 28,7 & $(24,0-33,3)$ \\
\hline 3. Bastante insatisfecho & 13 & 3,7 & $(1,7-5,7)$ & 12 & 3,3 & $(1,5-5,2)$ \\
\hline 4. Muy insatisfecho & 2 & 0,6 & $(0,0-1,4)$ & 1 & 0,3 & $(0,0-0,8)$ \\
\hline No sabe, no contesta & 2 & - & - & 7 & - & - \\
\hline
\end{tabular}

* Programa de Atención Dental Infantil y Juvenil.

\section{DISCUSIÓN}

En primer lugar, cabe destacar el alto índice de respuesta a los cuestionarios enviados, que oscila entre el 84 y el $96 \%$.

\section{Percepción del estado de salud y de la necesidad de tratamiento}

De manera coherente a los resultados del estudio sobre el nivel normativo de salud dental de los escolares de Navarra ${ }^{1}$, se constata una autopercepción positiva de salud dental. A más del 75\% de los padres de los de 6 y 12 años, y al 82,9\% de los niños de 14, les parece buena o muy buena y sólo entre el 6 y el $8 \%$ están insatisfechos o muy insatisfechos con su salud bucodental.

Asimismo, que aproximadamente una tercera parte de los de 12 y 14 años sienta que necesita algún tratamiento dental, y que mayoritariamente crean que es ortodóncico, es un resultado coherente con la prevalencia de maloclusión a esas edades ${ }^{1}$.
No obstante, sería interesante realizar un estudio más profundo para determinar qué relación existe entre la auto-percepción del estado de salud y los índices clínicos y entre las necesidades de tratamiento sentidas y normativas.

\section{Cepillado de dientes}

En cuanto a la frecuencia de cepillado dental es positivo que más del $61 \%$ de los escolares, a cualquier edad, diga cepillarse la boca dos o más veces al día (frecuencia estándar ideal $\left.{ }^{5,6}\right)$. No obstante, tratándose de una medida preventiva tan importante en el autocuidado de la salud bucodental ${ }^{7-9}$ sería deseable incrementar ese porcentaje en los próximos años.

Comparativamente, la frecuencia de cepillado dental de los escolares de Navarra es ligeramente mejor que en España ${ }^{10}$ y se encuentra en una situación intermedia con respecto a otros países de nuestro entor- 
no. Además, en todos los casos aparecen, como en Navarra, diferencias significativas por sexo $(\mathrm{p}<0,05)$, teniendo siempre mejor higiene dental las chicas (Fig. 1) ${ }^{11}$.

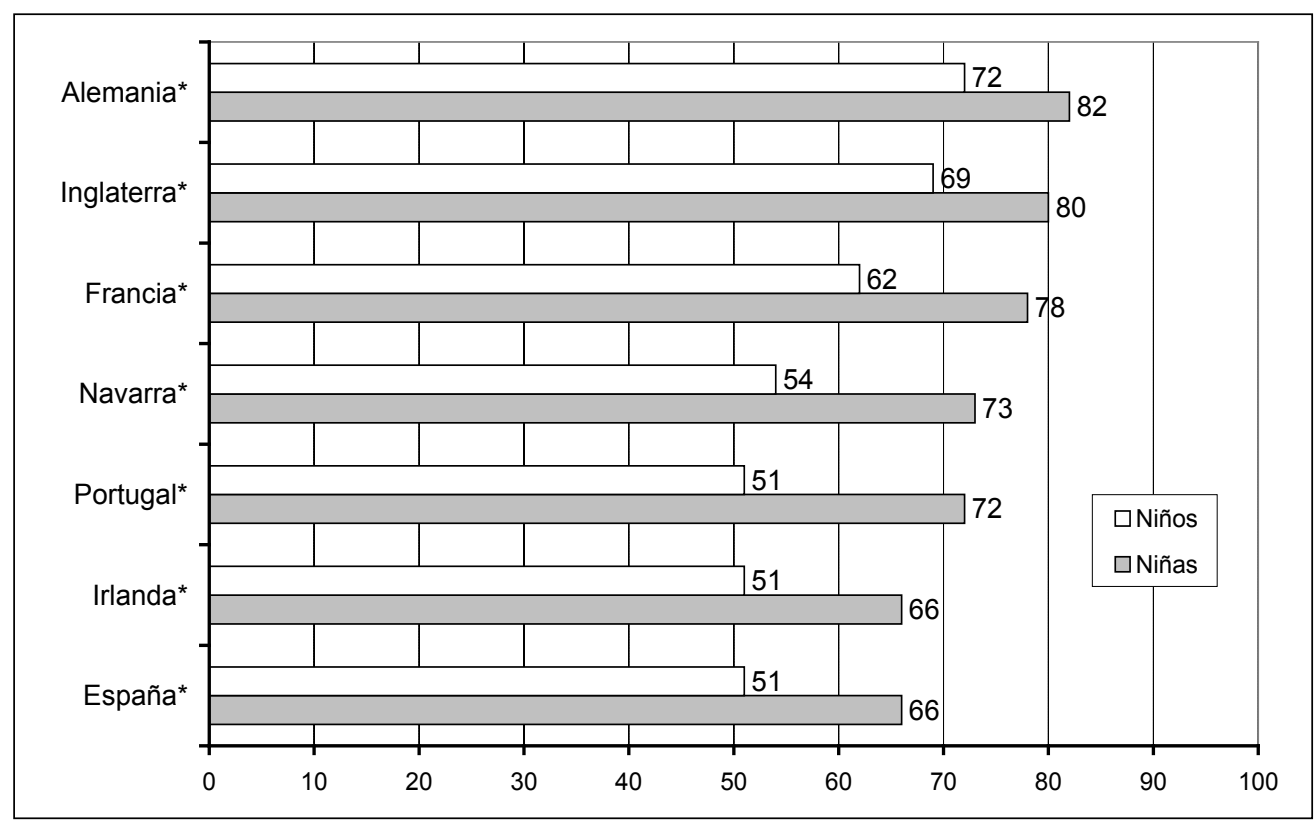

Figura 1. Porcentaje de niños/as de 13-14 años que se cepillan más de una vez al día, por sexo, en Navarra, España y algunos países
* Diferencia por sexo estadísticamente significativa $(\mathrm{p}<0,05)$.

\section{Asistencia al dentista}

La frecuencia de asistencia al dentista es muy alta, entre el 91,6 y $82,6 \%$ en el último año según grupos de edad, acudiendo la gran mayoría a un dentista PADI, sobre todo entre los más pequeños. En el grupo de 14 años el 51,1\% dice haber ido a un dentista PADI y el $31,1 \%$ a uno privado (Tabla 7). Hay una remarcable diferencia en estos datos entre el grupo de 14 años y los otros dos grupos de edad; a este respecto cabe señalar que los escolares de 14 años pudieron no interpretar adecuadamente la pregunta y parte de los que señalaron la respuesta "privado" en realidad era un dentista PADI (privado concertado) (ver metodología de cuestionarios en población y métodos). Por ello, en este grupo de 14 años, nos parece válido el dato global de asistencia pero no la discriminación entre PADI y privado.
Para próximos estudios convendría mejorar el sistema para determinar con mayor rigor la «asistencia al dentista durante el último año".

Por otra parte, es muy positivo que más del $75 \%$ de los padres de los escolares de 6 y 12 años califiquen como buenas o muy buenas las prestaciones ofertadas por el PADI. Sin embargo, sería conveniente investigar el motivo por el que cerca del $25 \%$ de los padres las califiquen como regular, mal o muy mal (Tabla 9).

\section{Influencia de las variables socio- demográficas}

Se ha analizado la asociación (en cada edad) de las variables sexo, residencia y NSE con la percepción en salud, frecuencia de cepillado y necesidades de tratamiento sentidas. Los hallazgos significativos 
$(\mathrm{p}<0, .05)$ de esas asociaciones son los siguientes:

- Con relación a la percepción de salud bucodental, sólo a los 14 años se asocia el NSE y la percepción (peor percepción en NSE bajo) (Tabla 4).

- Respecto al cepillado, las niñas se cepillan más que los niños a los 12 años (dato no mostrado) y a los 14 . Además, a los 14 años también es más frecuente en medio urbano (Tabla 6).

- En cuanto a las necesidades sentidas, a los 6 años hay más necesidades en NSE bajo (Tabla 2).

Además, algunas de estas diferencias significativas se corresponden con lo observado en el estudio del nivel normativo de salud ${ }^{1}$; concretamente:

- A los 14 años y NSE bajo, además de presentar peor percepción de salud, clínicamente la media de caries es mayor.

- A los 6 años y bajo NSE, además de sentir más necesidades de tratamiento, clínicamente presentan, en dentición temporal, mayor media de caries activa y menor índice de restauración.

\section{Evolución en asistencia al dentista y en hábitos de higiene}

A pesar de la posible dificultad que pudo tener la pregunta que medía la asistencia al dentista y aunque pudo haber confusión entre los encuestados en distinguir un dentista privado (por ejemplo, el ortodoncista) de uno del PADI concertado por el Servicio Navarro de Salud-Osasunbidea, puede afirmarse que:

- La asistencia al dentista es alta o muy alta.

- Desde 1992, el porcentaje de escolares que han ido al dentista durante el último año ha crecido considerablemente en los tres grupos de edad (Tabla 8), dato coherente con los índices globales de utilización del PADI desde 1991 hasta $2002{ }^{12}$.

Con relación a los hábitos de higiene, también cabe destacar el importante incremento ocurrido durante los últimos veinte años en el porcentaje de niños de 14 años que se cepillan los dientes más de una vez al día, que ha pasado del $23,4 \%$ en 1987 al 63,3 en 2007 (Fig. 2).

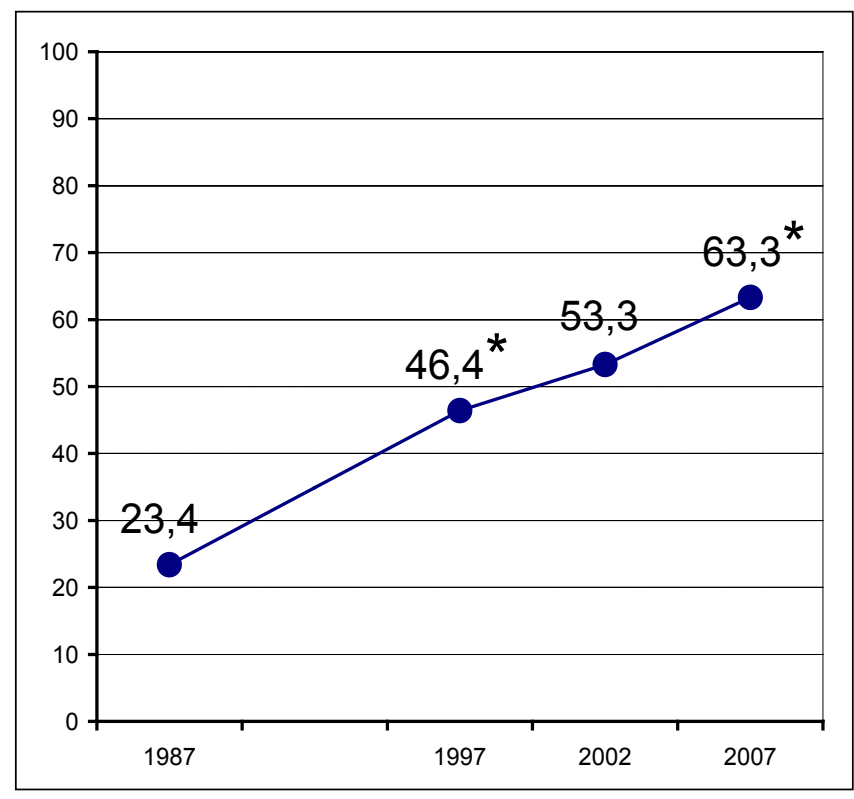

* Diferencia estadísticamente significativa con el año anterior (los IC-95\% no se solapan)

Figura 2. Evolución, desde 1987, del porcentaje de niños de 14 años que se cepillan más de una vez al día. 


\section{CONCLUSIONES}

1. En términos globales parece haber coherencia entre los datos clínicos de salud (baja prevalencia de enfermedad) y la auto-percepción de salud.

2. Asimismo, los datos clínicos de bajo nivel de enfermedad encontrados en las exploraciones clínicas son coherentes con las escasas necesidades de tratamiento expresadas por la misma población.

3. Los hábitos higiénicos siguen mejorando notablemente respecto a estudios anteriores, aunque deberían seguir haciéndolo.

4. La asistencia al dentista, después de quince años de constante incremento, puede calificarse como muy alta.

5. La higiene bucodental es algo mejor en las chicas de 12 y de 14 años.

6. La pertenencia a un nivel socio-económico bajo sólo genera peor autopercepción de salud a los 14 años (no al los 6 ó 12) y más necesidades de tratamiento sentidas a los 6 (no a los 12 ó12 ó 14). El nivel socioeconómico bajo tampoco genera diferencias significativas en cuanto a la frecuencia de cepillado dental en ninguno de los grupos de edad estudiados.

\section{Agradecimientos}

Nuestro agradecimiento a todos los escolares de la muestra seleccionada y a sus familias por su consentimiento y participación. Asimismo, a los centros escolares por la autorización a utilizar sus instalaciones. Finalmente, al Departamento de Educación del Gobierno de Navarra por su colaboración.

\section{BIBLIOGRAFÍA}

1. Cortés Martinicorena FJ, Artázcoz Osées J, Bravo M, González Rodríguez P, Rosel Gallardo E, Asenjo Madoz MA, et al. La salud dental de los niños y adolescentes de Navarra, 2007 (4⿳亠口了 edición). An Sist Sanit Navar. 2009; 32(2): 199-215.

2. Abad Vicente FJ, Cortés Martinicorena FJ. Estudio Epidemiológico de la salud bucodental en la población escolar 1987. Dirección de
Atención Primaria. Departamento de Salud. Gobierno de Navarra. 1989.

3. Cortés J, Doria A, Martínez I, Asenjo MA, Sainz de Murieta I, Cuenca E. Prevalencia de caries dental entre la población infantil de Navarra, 1997. Arch Odontoestom Prev Comunit 1998; 14: $640-646$

4. Doria-Bajo A, Cortés-Martinicorena FJ, AsenjoMadoz MA, SÁinz de Murieta-Iriarte I, RamónTorrell JR, Cuenca-Sala E. Hábitos de higiene oral en los escolares de Navarra, 2002. Arch Odontoestom Prev Comunit 2003; 19: 515522 .

5. Junco Lafuente MP, Baca García P. Métodos de control de la placa bacteriana. En: Cuenca Sala E, Baca García P, editores. Odontología preventiva y comunitaria. Principios, métodos y aplicaciones. $3^{\mathrm{a}}$ edición. Barcelona: Masson, 2005: 87-104.

6. LöE H. Oral hygiene in the prevention of caries and periodontal disease. Int Dent $\mathrm{J}$. 2000; 5: 129-139.

7. Marinho VCC, Higgins JPT, Sheiham A, Logan S. Cremas dentales fluoradas para prevenir caries dentales en niños y adolescentes (Revisión Cochrane traducida). En: La Biblioteca Cochrane Plus, 2008 Número 4. Oxford: Update Software Ltd. [consultado 22-04-2009]: Disponible en: http://www.update-software. com (Traducida de The Cochrane Library, 2008 Issue 3. Chichester, UK: John Wiley \& Sons, Ltd.)

8. Pine CM, Curnow MM, Burnside G, Nicholson JA, ROBERTS AJ. Caries prevalence four years after the end of a randomised controlled trial. Caries Res 2007; 41: 431-436.

9. Sutcliffe P. Oral cleanliness and dental caries. En: Murray JJ, Editor. The prevention of dental disease. 2 edición. Oxford: Oxford University Press, 1989: 200-217.

10. Encuesta Nacional de Salud del Instituto Nacional de Estadística de España. [consultado 2204-2009]: Disponible en: http://www.ine.es/jaxi/ menu.do?L=0\&type=pcaxis \&path $=\% 2 \mathrm{Ft} 15 /$ p419\&file=inebase

11. Health behaviour in school-aged children (HBSC) 2005/2006. WHO/OMS. [consultado 22-04-2009]: Disponible en: http://www.euro. who.int/Document/E91416_Ch2_3.pdf

12. Cortés FJ, Ramón JM, Cuenca E. Doce años de Pprograma de Aasistencia Ddental ilnfantil (PADI) en Navarra (1991-2002). Utilización e indicadoresindicadores de salud. An Sist Sanit Navar. 2003; 26: 373-382. 